\title{
THE GENERAL MANAGEMENT OF THE PHTHISICAL PATIENT FROM THE PHYSICIAN'S STANDPOINT
}

\author{
By L. E. Houghton, M.A., M.D. \\ Consulting Physician, Harefield Hospital
}

In the management of pulmonary tuberculosis, as in military affairs, it is possible to differentiate between strategy and tactics. By means of chest radiography the field of operations can be mapped out with great accuracy, the disposition of the opposing elements be closely assessed and their probable behaviour often be anticipated. This, together with a knowledge of the terrain, enables a plan of campaign to be evolved and action decided upon by a certain measure of remote control. This has sometimes led to the disparaging comment that chest physicians treat, not patients, but $\mathrm{X}$-ray films. But such criticism takes no account of the physician's major functions-the tactical approach to the patient in the clinic and at the bedside.

\section{The Round Focus}

For example a patient who is quite symptom free may be discovered on radiography to have a localized opacity or 'round focus' in his lung. This will lead to a discussion among his physicians, centred very largely upon radiological appearances, as to the aetiology and probable behaviour of the lesion. Its size, consistency and position will all be taken into account. The patient's age and sex, his family history and his contacts, his work and his habits will come under review. Is this a recently developed shadow or is it a relic of the long past, perhaps a healed primary focus? Does it contain calcium? It may be decided that it does, in fact, represent a reaction to the tubercle bacillus and its behaviour may well depend not only upon its size but upon the way in which it has developed. It may represent an adult primary focus or it may be a localized mass of granulation tissue (a tuberculoma); it may be a patch of atelectasis secondary to a tuberculous endobronchitis. At the back of the physician's mind lurks the possibility that it may be a neoplasm. The surgeon is in a strong position when he says "When in doubt take it out.' But awaiting the physician in his consulting room is an apparently healthy young man or woman. Already the opacity in this patient's lung has cast a shadow across his life. He has been recalled for a large film, he has had tomograms taken, he has had blood taken for E.S.R. and a laryngeal swab. Obviously there is something wrong. It may well be that the physician has by no means made up his mind about the prognosis, but no such doubts must be communicated to the patient. Unless he has decided upon resection he must come down firmly on the side of reassurance. An explanation of the ubiquity of the tuberculous infection and the common finding of scars in the lungs of healthy people should be his theme and he should emphasize the routine nature of a check up in such cases. It must be pointed out that it is usual to repeat the check up periodically for a time. But there must be no dubious shaking of the head concerning the future. The doctor must take the risk of being wrong rather than inflict months of anxiety upon the patient and his relations. It is, indeed, an unfortunate teature of mass radiography that it has bred much avoidable neurosis.

\section{Active Tuberculosis}

In the case of the round focus discovered by routine X-ray, the management of the case lies broadly between prophylactic resection and prolonged observation. Putting the patient on light work, admitting him to hospital or administering drugs are unlikely to affect the issue. But when open tuberculosis has been diagnosed the problem is quite a different one. Active treatment must begin at once. Again, however, the doctor's first interview with his patient is the most important of all. The impact of diagnosis can be softened by the logical explanation of his past symptoms and malaise and the assurance that under treatment he will return to a much higher standard of general 
health. The resentment that many patients feel on diagnosis often causes them to seek a scapegoat for their misfortunes and the doctor who, months ago, may have prescribed a cough mixture for bronchitis or a tonic for debility, receives full measure of hostile comment. The resentment which patients feel at the diagnosis and the necessity for them to submit to prolonged sequestration trom normal life is often reflected in their attitude towards treatment. The less discerning trequently assume the attitude of martyrs submitting unwillingly to a régime which is chiefly the concern of the medical authorities. Because of this understandable attitude doctors and nurses treating the tuberculous must not expect to receive an expression of appreciation which the patients certainly do not feel towards them. Such lack of moral support from one's patients, even from the most successfully treated, is one of the few discouraging features of work in this field of medicine.

It is as well, at this first interview, to ask the patient if he himself can account for the onset of his trouble. He will often associate it with specific periods of stress or exposure to adverse conditions, and in this he may well be right. This is indeed a good starting point for rehabilitation and it will give the patient confidence that he will be able to avoid similar circumstances on his return to his normal mode of life. Such a discussion will also help to establish good rapport because the patient will feel that the doctor really understands his particular case. The physician working in a hospital or sanatorium has an advantage in this way because he sees his patient almost daily for many months and while steering him through the crisis of his illness, comes to know him very well. In the past when active therapy was minimal and community organization was paramount in treatment, the Medical Superintendent could act in the capacity of counsellor to all of the patients in the sanatorium. But now that treatment is individual and intensive the bedside tactics should be left to the ward doctors, and the physician in charge should direct strategy from a position of vantage. The patient should only have one doctor at a time.

The appointment of highly qualified and keen clinicians to clinic posts, with a considerable number of beds under their care, has perhaps tended to change the emphasis in chest clinic work from prevention and after-care to bedside treatment. It would seem that there is scope for the appointment of a second permanent physician unless the long-term after-care of tuberculous patients is to be largely undertaken by a succession of registrars. This latter arrangement is disconcerting to the patient, who may never see the :same doctor twice, but there is an 'obvious limit to the amount of work that the chief physician can undertake.

\section{Major Surgery}

The association of physicians and surgeons in the treatment of pulmonary tuberculosis is close and rewarding. There is, at times, a divergence of opinion upon expediency and timing, but more often, a convergence of approach to the final problem, which is the prevention of relapse. It is a good working rule for the physician to decide which of his patients will benefit from major surgery and to present them to the surgeon at the right time; but the surgeon alone can decide whether or not he wishes to operate and what procedure he considers to be appropriate. As has been said, the function of major surgery is the prevention of relapse, and this implies that the patient should have been brought to a point where no further improvement is to be expected from medical treatment, and yet some active, or potentially active, focus of disease remains. The presence of an open cavity with or without positive sputum is a clear indication for surgical intervention. A much more difficult situation is presented by the cavitated lesion which resolves after a successful course of chemotherapy leaving no tomographic trace of excavation and negative laryngeal swabo culture. Knowledge of the prognosis of such cases will remain obscure until an adequate seriesog treated conservatively, has been followed up for a number of years. In the absence of tomographic evidence of cavitation, a bronchogram may show residual bronchiectasis in an area where there has been extensive infiltration before chemotherapy. If this abnormality is limited to a lobe or a segment, the surgeon may feel encouraged to resect that portion of the lung. Certainly if a patient with no tuberculous history but having a background of frequent chest symptoms, were to present with such definite radiological evidence of local bronchiectasis, resection would be the treatment of choice. But the ectatic and distorted bronchi resulting from healed tuberculosis do not tend to become secondarily infected. In the absence of a positive sputum, therefore, the demonstration of such lesions by bronchography is not in itself an indication for resection. In the presence of a persistently positive sputum or sputum culture, however, a bronchogram may be of considerable value to the surgeon in localizing the diseased area. Excellent bronchograms, with the advantage of rapid clearing, can be obtained by the use of sulphanilamide suspended in lipiodol.

\section{Timing of Surgical Intervention}

Reference has been made to the timing of surgical procedures. There are three main con-

\section{.}


siderations; radiological appearances, sputum results and the patient's general condition. If the radiological appearances are deteriorating, surgery is probably out of the question; if they are static, surgery may be indicated. A persistently positive sputum should obviously encourage surgical intervention. It is tempting in some cases where sputum remains positive in the presence of localized lesions to continue with repeated courses of chemotherapy, but the surgeon should not be called upon in the end to operate upon a patient whose organisms have become resistant to the antibiotics.

One of the major functions left to the physician in the field of thoracic medicine is the assessment of 'general condition.' In tuberculosis the problem is seldom one of cardiovascular function but rather of respiratory reserve and moral fortitude. The assessment of respiratory function by bronchospirometry has been considerably advanced in recent years and much information can now be expressed in a factual manner. Of particular value in tuberculosis is the differential evaluation of function in either lung. Upon such an assessment may be determined the feasibility of pneumonectomy. But the procedure remains a little complex and differential bronchospirometry is definitely harassing to the patient. Alternatively simple vital capacity assessments reveal little of value. On the whole it is probably true to say that apart from the exceptional case the physician should be able to make his own assessment trom clinical examination and from radiological appearances. Generalizations are known to be dangerous but where respiratory function is in doubt thoracoplasties are safer than resections and post-operative shock is often more pronounced after plombage.

The assessment of 'moral fortitude' is not without importance. It is best considered by an unhurried conversation with the patient upon matters not necessarily associated with his disease. The apparently phlegmatic patient is not ipso facto the best candidate for the stress of a three-stage thoracoplasty. The experienced ward sister is, however, often a good judge not only of the patient's reaction to a surgical programme but also of the right time for intervention.

It is true to say that chemotherapy has added very greatly to the numbers of cases in which major surgery is feasible, but surgery is not an end in itself and it is still the function of the physician to avoid thoracotomy if this will not prejudice the patient's future. A major operation upon the chest wall and upon the lung itself is a serious catastrophe for a young man and even more so for a young woman. To recognize this fact is not to decry the life-saving nature of such operations. Under modern conditions operative mortality is low (albeit not negligible), but physical and psychological trauma is very considerable. The physician's task is made more difficult by the current belief among patients that surgery is the only certain method of cure and that the sooner it is over and done with the better. Moreover one of his chief alternatives to major surgery, pneumothorax, has suffered eclipse during recent years.

\section{Pneumothorax}

There are good reasons why some physicians are now reluctant to induce a pneumothorax. The treatment has been seriously abused in the past for the ease of induction and the lack of alternative treatment resulted in wholesale ' crash pneumothoraces' without adequate bed rest and without regard to the dangerous mechanical situations engendered. Ill advised inductions were followed by inexpert management in refill clinics of unwieldy size. The overall result was a very high complication rate and it is probable that in the last two decades much more harm than good has resulted from this form of treatment. For those, however, who by a painful process of trial and error have become aware of the limitations and potential dangers of pneumothorax, the treatment remains of the greatest value in selected cases. The state of the bronchi is all important in the progress and management of pulmonary tuberculosis and one of the chief events to be avoided in pneumothorax is bronchial occlusion. ${ }^{1}$ The effect of streptomycin in conditioning patients for pneumothorax is not altogether clear. As is well known, one of its most obvious actions is upon the bronchial mucosa where it brings about rapid healing of the tuberculous process. The rapidity of healing under streptomycin and the subsequent cicatrization may promote occlusion of the smaller and terminal bronchi, and this in turn gives rise to patchy atelectasis. If the areas had not been coincidentally sterilized by the drugs this result would certainly be unsatisfactory. In any event the radiological appearance of discrete nodules in the affected area is somewhat discouraging to the collapse therapist and suggests resection as the appropriate treatment. Thus streptomycin, by the rapidity of its action, forestalls the induction of pneumothorax. If this results in an increasing recourse to major surgery there might well be an indication for pneumothorax induction in appropriate cases soon after chemotherapy has started. The desirability of avoiding not only fibrosis but also destructive inflammatory changes during the active phase of pulmonary tuberculosis suggests the use of cortisone or ACTH in combination with chemotherapy as an attractive possibility. As these 
hormone:s also appear to have a very definite effect in ameliorating toxaemia in active phthisis it is conceivable that they may prove to have some overall clinical value. A limited trial has been carried out and the results are in the press, but a great deal more work would be required before the use of these hormones in tuberculosis could be regarded as valuable or justifiable.

\section{Rehabilitation}

The patient is liable to emerge from his tuberculous ordeal in a state of suspended animation. He has been told what he must not do and what he is not fit to do for many months. He has seen some of his associates succumb to the disease and others return for further treatment. It has been suggested to him that he is not really suitable to consort with his fellow men-perhaps even his wife and children - and he may have had quite large and significant portions of his body removed. As soon as he has struggled to his feet he wants, not unnaturally, to go home and get away from it all. $\mathrm{He}$ will then make periodic, apprehensive, visits to his clinic and sooner or later will be told that he can begin ' light work.' It is very unlikely, however, that he is fit physically or psychologically to start looking for normal employment in industry.

Some sanatoria fortunately adhere to the time honoured regime of graded rest followed by graded exercise before discharge. Many of the larger hospitals and sanatoria are, however, so much preoccupied with the high drama of thoracic surgery that the time spent upon the restoration of physical and psychological well being is often much curtailed. It is a sound practice to insist upon 12 weeks of bed rest after a major thoracic operation for tuberculosis to be followed by 12 weeks of graded rehabilitation. The atmosphere of a busy surgical unit is not entirely suitable for the management of the restless convalescent, and strict hospital routine is neither desirable nor necessary during this period. For these reasons there is an advantage in undertaking the final stages of treatment at a separate unit. An example of such a centre is Grim's Dyke Rehabilitation Unit at Harrow Weald, Middlesex.

Here 50 beds are provided for ambulant and $\frac{3}{\sigma}$ semi-ambulant male patients in a converted $\stackrel{\varrho}{c}$

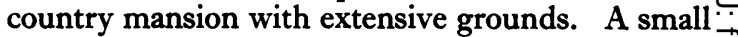
nursing and administrative staff is augmented by an outdoor work supervisor, a woodwork instructor and a patients' warden. Patients are encouraged to undertake an increasing amount of outdoor work under careful clinical supervision. During in- $\widehat{\Phi}$ clement weather they are given indoor occupations. 0 For the last month of the three months' course they make articles for themselves in the woodwork $\overrightarrow{0}$ shop. It is insisted that patients shall keep to $\overrightarrow{\vec{H}}$ regular hours of work, but apart from this a good $\omega$ deal of personal freedom is allowed. No restric- 8 tion is placed upon leaving the centre in the 3 . evenings up to 9 p.m., and alternate week-ends are spent at home. In this way patients are en- $-\infty$ couraged to return to the rhythm of regular work $\stackrel{\vec{\perp}}{+}$ while under observation and at the same time theyin are regaining a footing in their home environment. The results are clearly seen in the return ofo physical fitness and confidence. Before discharge arrangements are made by the warden for a return $\subseteq$ to previous employment or alternatively a new job is found. It is considered essential that a specific $\vec{D}$ date for re-starting work shall be arranged befooec the patient leaves the centre.

Many of the men make use of their stay t.ㅎㅇ응 undertake courses of study and others are passed on to a Government Training Centre. It is essential for the success of this scheme that onlyo those patients who have successfully completed $\stackrel{\complement}{\complement}$ active treatment shall be admitted to the centre. $\overrightarrow{\vec{A}}$ It is not possible, therefore, to admit cases with positive sputum or chronic open lesions for it is assumed that all admissions will pass on at the end of the course to full employment in industry.음 It is a satisfying thought that an ever-increasing. percentage of patients treated for pulmonaryo tuberculosis are able to comply with this standard:

REFERENCE

I HOUGHTON, L. E. (1950), Tubercle, 31, 3.

\section{RUTHIN CASTLE, NORTH WALES}

A Clinic for the diagnosis and treatment of Internal Diseases (except Mental or Infectious Diseases). The Clinic is provided with a staff of doctors, technicians and nurses.

The surroundings are beautiful. The climate is mild. There is central heating throughout. The annual rainfall is 30.5 inches, that is, less than the average for England.

The Fees are inclusive and vary according to the room occupied.

For particulars apply to THE SECRETARY. Ruthin Castle, North Wales.

Telegrams: Castle, Ruthin.

Telephone: Ruthin 66 